\title{
Théorie constitutive : reconnaissance, éthique et politique dans les relations internationales
}

\section{Mervyn Frost}

\section{(2) OpenEdition \\ 1 Journals}

Édition électronique

URL : http://journals.openedition.org/conflits/18472

DOI : $10.4000 /$ conflits. 18472

ISSN : $1777-5345$

Éditeur :

CCLS - Centre d'études sur les conflits lilberté et sécurité, L'Harmattan

Édition imprimée

Date de publication : 26 décembre 2012

Pagination : 37-46

ISBN : 978-2-336-00248-4

ISSN : 1157-996X

Référence électronique

Mervyn Frost, «Théorie constitutive : reconnaissance, éthique et politique dans les relations

internationales », Cultures \& Conflits [En ligne], 87 | Automne 2012, mis en ligne le 26 décembre 2013, consulté le 30 mars 2021. URL : http://journals.openedition.org/conflits/18472 ; DOI : https://doi.org/ $10.4000 /$ conflits. 18472 


\section{Théorie constitutive : reconnaissance, éthique et politique dans les relations internationales 1}

\section{Mervyn FROST}

Mervyn Frost est Professeur de Relations Internationales à King's College (Londres). Il est depuis 2007 le directeur du département des études polémologiques (War Studies). Ses travaux portent sur les questions éthiques en relations internationales.

$\mathrm{D}$ ans un article intitulé «La reconnaissance entre les États : sur le substrat moral des relations internationales » Axel Honneth relève que dans le langage usuel, on parle souvent des États comme cherchant le respect et la reconnaissance de la part d'États étrangers. Nous comprenons aisément les États lorsqu'ils font valoir leur droit à une reconnaissance dont on les prive. Honneth souligne que «nous convenons volontiers que le comportement des dirigeants politiques de la Palestine, par exemple, ne peut être compris sans prendre en compte de telles aspirations à une reconnaissance; que le gouvernement russe réalise de gros efforts pour imposer le respect aux pays occidentaux ou bien que pendant les mandats de Bush, les gouvernements d'Europe occidentale ont usé de relations et manœuvres diplomatiques pour renouveler le respect de leur allié américain ». Pourtant, lorsque nous nous tournons vers la discipline des Relations Internationales (RI), nous constatons que la notion de reconnaissance n'y joue pas un rôle central. Les théories qui prédominent décrivent plutôt des États souverains indépendants agissant comme des acteurs cherchant à maximiser leur pouvoir dans le but de poursuivre leurs intérêts. Les États opéreraient de manière instrumentale. Contre cette conception, Honneth cherche à élaborer une approche des RI davantage centrée sur la notion de reconnaissance. Pour lui, il faut considérer sérieusement le fait que les populations des États souverains veulent que les gouvernements leur garantissent le respect dû à leurs réalisations : leur histoire, leur constitution et surtout, leur mode de vie en général. En bref, Honneth attire notre attention

1. Texte traduit de l'anglais par François Gleize-Colombera. 
sur la lutte pour la reconnaissance qui se joue au sein des affaires internationales.

Honneth avance que le type de reconnaissance qui importe dans les relations internationales implique beaucoup plus qu'une simple reconnaissance de la conformité juridique d'un État, sur le plan du droit international. La reconnaissance juridique se résume simplement, selon lui, par le constat de conformité que peuvent établir les juristes qui ne cherchent à répondre qu’à la question suivante : «Cet État remplit-il, oui ou non, les conditions juridiques telles qu'elles sont définies aujourd'hui? ». La notion de reconnaissance chez Honneth implique plus que ce noyau juridique. Ce que l'on recherche, c'est une compréhension de ce à quoi les personnes accordent de la valeur au sein de leur propre société, à l'intérieur d'un État particulier. Cette appréciation de leur société comprend le sens de son histoire, de ses valeurs éthiques ainsi que le sentiment d'un progrès accompli. Ce sont ces éléments que les individus souhaitent voir respecter par les autres États. De la même façon, les autres États auront leurs propres conceptions des éléments de leur société devant être respectés. L'idée centrale de l'argumentation d'Honneth est que l'État pense que son intérêt national comprend cette composante de reconnaissance et que cette dernière devrait lui être accordée au titre d'agent de la sphère internationale. Une prise en compte sérieuse de la lutte pour la reconnaissance offre, selon Honneth, un ensemble d'explications bien plus convaincant de la manière dont fonctionne la politique internationale. En effet, la lutte pour la reconnaissance implique ce qu'il appelle « les politiques de reconnaissance». Dans cet article, je tiens à développer et à examiner de manière critique cette idée.

Je suis d'accord avec l'argument principal avancé par Honneth. Le concept de reconnaissance est un élément central pour toute bonne compréhension des affaires internationales. Cependant j'entends montrer que cela est lié davantage à la notion elle-même. En particulier, je souhaite examiner de quelle manière la reconnaissance est intégrée dans deux pratiques internationales spécifiques et comment elle ne peut être comprise qu'en leur sein. Suite à cela, je détaillerai un aspect souvent négligé : la reconnaissance réciproque dans les affaires internationales. Ce qui est particulièrement important ici est que la reconnaissance réciproque suppose une notion d'égalité éthique. Les pratiques qui fondent ce genre de reconnaissance sont donc des pratiques supposant une égalité entre les agents participants. De plus, je soulignerai à quel point, dans le cadre actuel des pratiques internationales, la lutte pour la reconnaissance est liée à la valeur éthique de liberté individuelle. Par liberté individuelle, j'entends la liberté de chaque participant d'agir et de reproduire consciemment ces pratiques. Finalement, je m'attacherai à la façon dont nous devons comprendre la guerre en relation avec la liberté et la reconnaissance réciproque. C'est seulement quand nous comprendrons comment l'octroi (ou le refus) de reconnaissance fonctionne dans nos deux pratiques mondiales fon- 
damentales, au sein de la société civile mondiale et de la société des États souverains, que nous aurons une bonne compréhension de ce que les décideurs en politique étrangère ont à défendre, parfois au moyen la force militaire.

La notion générale de reconnaissance ne peut être élucidée dans la pratique des RI, car elle est sous-déterminée. Prenons des exemples impliquant une notion rudimentaire de la reconnaissance. Afin de préparer un discours politique durant une course électorale, un candidat à la présidence doit savoir à quel moment il le prononcera. Afin d'écrire une "tribune libre » en Relations Internationales, un auteur doit identifier un auditoire auquel il doit s'adresser. Afin de déclarer la guerre, un Premier ministre doit repérer le lieu et la manière appropriés pour le faire. Afin de commencer une conversation sur la politique mondiale avec mon lecteur, j’ai besoin de savoir que le moment est opportun pour l'initier. Pour réaliser ce que je fais maintenant, $j$ 'ai dû reconnaitre un certain nombre de choses. Bref, l'idée de reconnaissance, prise en elle-même, est trop générale pour nous dire grand chose sur quoi que ce soit.

Quand je dis, à la suite d'Honneth (qui lui-même suit Hegel), que la reconnaissance est importante pour l'étude de la politique mondiale, je ne parle pas de n'importe quel type de reconnaissance, mais seulement d'une forme particulière de reconnaissance réciproque qui participe des pratiques constitutives des rapports internationaux. Nous devons donc saisir comment s'engagent les processus de réalisation de cette reconnaissance particulière. Ce que nous devons comprendre n'est pas simplement un ensemble d'expressions idiomatiques de la reconnaissance issu des recherches d'Hegel - par exemple : comment être un maître qui reconnaît un esclave et comment un monarque reconnaît un sujet ; ou bien comment être un sujet reconnu d'un monarque, ou encore comment un oligarque reconnait ceux sur lesquels il exerce son pouvoir, et ainsi de suite. De nombreuses formes de reconnaissance peuvent sans doute être identifiées dans les rapports internationaux, mais celles-ci ne nous paraissent pas cruciales. En revanche, deux formes spécifiques de reconnaissance réciproque sont au cœur de nos rapports internationaux contemporains. La première est la reconnaissance accordée par les États libres à d'autres États libres. Dans cette relation, les États se reconnaissent mutuellement comme libres et égaux dans le sens d'une égalité juridique formelle. La seconde est la reconnaissance accordée par les titulaires de droits individuels envers d'autres titulaires de droits. Dans cette relation réciproque, les parties reconnaissent, une fois de plus, l'autre comme formellement libre et égal.

La première forme de reconnaissance réciproque se situe dans la pratique de la souveraineté des États à laquelle nous participons tous. Dans ce «nous » j'inclus l'ensemble des individus quel que soit l'endroit où ils se trouvent. La seconde se trouve dans les rapports de la Société Civile Mondiale (SCM), c'est-à-dire dans les rapports entre les titulaires des droits de l'Homme. Parce 
que la reconnaissance demandée et accordée selon ces pratiques est très spécifique, nous devons préciser avec soin ses caractéristiques analytiques.

Dans ces pratiques de reconnaissance réciproque, les acteurs ne reconnaissent pas chez l'autre la préexistence d'une caractéristique appelée "liberté ». Le type de liberté en jeu ne préexiste ici aucunement à la reconnaissance d'autrui comme libre. Au contraire, la liberté s'établit au moyen d'une reconnaissance mutuelle ou à chaque fois que des agents se reconnaissent libres dans un rapport social. Ainsi, par exemple, l'État souverain X (disons l'Afrique du Sud) n'est libre que dans la mesure où il est reconnu comme tel par des États eux-mêmes libres dans leurs rapports de souveraineté. Une entité non reconnue politiquement ne peut unilatéralement se proclamer comme libre car, sans la reconnaissance des autres États, elle ne sera pas estimée comme telle dans sa pratique des relations internationales. La liberté n'est pas une chose, une abstraction, qui peut s'acquérir par un État indépendamment de la pratique qui fait que les Etats se reconnaissent libres entre eux.

De la même façon, des États qui sont considérés « libres » par des États qui ne sont pas eux-mêmes reconnus comme libres ne deviennent pas libres pour autant. Dans les rapports entre États libres, ce type de déclaration n’a aucun effet. Quel que soit le nombre de déclarations émanant de partis politiques, de mouvements religieux ou autres groupes de pression, rien de cela ne suffira. Les pratiques constitutives fonctionnent par détermination de l'identité de ses participants.

Tout cela peut sembler un peu opaque. Permettez-moi d'éclaircir mon propos par un exemple qu'Hegel utilise dans le chapitre 7 de la Phénoménologie de l'Esprit. La reconnaissance réciproque entre maitres et esclaves n'est pas établie librement. Les esclaves peuvent être obligés de dire qu'ils reconnaissent le maître comme libre, mais ce qu'ils font ne constitue pas le maître comme étant libre. L'élément crucial d'une réciprocité est absent. Ceci peut être illustré par l'exemple de la politique sud-africaine. Lorsque le gouvernement d'Apartheid a créé ce qu'on appelle les «bantoustans », il a affirmé qu'il s'agissait d'États libres et souverains. Toutefois, ce type de reconnaissance n'a pas établi une liberté réciproque car il s'agissait d'une relation maître/esclave. Les bantoustans étaient une création du gouvernement sudafricain au sein de l'État sud-africain, ils restaient totalement dépendants de ce dernier. Il ne s'agissait pas d'acteurs libres ayant la possibilité d'adopter une politique hostile envers leur créateur. Dans la pratique des relations entre des États autonomes, ce qui est primordial pour des acteurs cherchant à constituer autrui comme libre est qu'ils se reconnaissent réciproquement comme tels et qu'ils s'accordent entre eux un espace de liberté. Pour être libre au sein de ces relations, il faut être libre de prendre des décisions pouvant être contraires aux valeurs et aux attentes des autres États, tout en ne contredisant pas les valeurs de base de la liberté. 
Il existe une autre façon d'exprimer cette revendication. Pour moi, vous reconnaître comme une personne libre exige que je crée un espace éthique dans lequel vous pouvez aller de l'avant et agir en tant que personne autonome. Je ne peux me contenter d'affirmer que vous êtes libre, puis exiger de vous un comportement que je dicterai. Si le respect que je porte à votre liberté est réel, je dois valoriser les choix que vous faites, même si je ne les apprécie peut-être pas. Imaginez une puissance impériale donnant théoriquement la liberté à une ancienne colonie (les îles Malouines par exemple) mais qui, dans les faits, agit à l'opposé. Elle porterait atteinte et réduirait à néant les politiques adoptées par le gouvernement « autonome » de cet «État». Ce ne serait certainement pas rendre la liberté à cette colonie. Pour reconnaître un acteur comme libre, il faut lui reconnaitre un espace social légitime de différence, un espace dans lequel il peut s'opposer aux décisions des autres parties. En effet, il faut reconnaître qu’à la limite, il existe une réelle possibilité pour un État de ne pas reconnaître ni d'approuver les choix d'un autre. En d'autres termes, par l'octroi de la liberté, on donne à autrui le pouvoir de porter un jugement sur nos propres décisions. Notre propre liberté dépend de ce que les autres nous accordent. Seuls ceux qui sont libres dans ces rapports peuvent octroyer un statut d'autonomie. La liberté ne peut être acquise par l'usage de la force. La force y est vaine.

Permettez-moi de démontrer ce point en me référant à un autre comportement, celui de l'amitié entre les êtres humains. Un individu ne peut forcer autrui à être son ami. Un ami est quelqu'un qui a accepté une ouverture amicale au sein d'un rapport social. À une ouverture amicale est espérée une réaction sans contrainte. Un ami est celui qui a donné librement son amitié à autrui. Dans cette relation, les participants apprennent à se présenter comme ouverts à une amitié. Quelle que soit la forme de reconnaissance obtenue par l'utilisation de pots-de-vin, d'incitations, de menaces et d'autres méthodes de ce genre, celle-ci n'apportera pas la reconnaissance qui est propre à l'amitié.

Dans les rapports mondiaux de souveraineté, grâce au système de reconnaissance réciproque (codifié et pris en charge de nos jours par le droit international), les États s'accordent un ensemble équivalent de libertés ou le droit de poursuivre librement les politiques de leur choix. Pour faire référence à cet ensemble de relations, nous utilisons habituellement le terme générique de «souveraineté ». C'est ce qui correspond à ce que les États réalisent par leur participation aux relations internationales. Il ne s'agit pas d'une chose que n'importe quelle entité politique peut obtenir indépendamment du mode de reconnaissance réciproque propre à la pratique des États.

À la lumière de cette analyse, me voilà maintenant plus à même de discuter les résultats présentés par Honneth. L'élément central de sa réflexion est que la lutte pour la reconnaissance est au fond une lutte politique entre les États. Cette conception politique de la lutte veut que chaque État tente de 
gagner auprès des autres États le respect de sa culture, de son mode de vie, de son histoire commune, et de ses engagements éthiques. Je pense qu'Honneth a en tête la lutte d'un État (supposons pour l'instant qu'il s'agisse des ÉtatsUnis) pour obtenir des autres une appréciation de ce qu'il représente, de sa constitution ainsi que de ce qu'il a historiquement accompli. On pourrait alors considérer les États-Unis comme étant engagés dans une lutte pour que la Chine prenne conscience de sa vision de la démocratie ainsi que des réalisations culturelles et historiques de son peuple. Inversement, la Chine pourrait tenter d'impressionner les États-Unis et d'autres pays par sa vision alternative du système démocratique ainsi que de sa culture et ses réalisations historiques. Dans la version de la lutte pour la reconnaissance d'Honneth, les États s'efforcent d'obtenir des autres États la reconnaissance de la valeur de leurs modes de vie. Dans la mesure où ils réussissent, ils auront obtenu la reconnaissance qu'ils désirent.

Bien que je reconnaisse l'importance de la lutte pour la reconnaissance entre États que décrit Honneth, je tiens à insister sur une forme de reconnaissance qui lui est, analytiquement parlant, antérieure. J'aimerais porter à l'attention du lecteur la présence d'un substrat de reconnaissance mutuelle qui est une condition constitutive de la lutte politique dont parle Honneth. Dans les rapports de reconnaissance mutuelle que j’ai soulignés auparavant, les États ne mettent pas tout d'abord en avant la reconnaissance de leurs modes de vie propres. Au contraire, ils se reconnaissent mutuellement comme étant le type d'entité habilitée à agir de la sorte en raison d'un rapport antérieur stipulant la forme de cette reconnaissance. Avant même de pouvoir commencer à rechercher la reconnaissance de leurs réalisations culturelles, ils doivent reconnaître une situation de pratiques partagées, c'est-à-dire qu'ils doivent accorder à autrui les conditions de sa liberté (un statut d'acteur autonome), base pour conduire et développer son propre mode de vie. Dans cette phase préalable de reconnaissance mutuelle, les États s'accordent réciproquement un ensemble légitime de libertés dont l'usage leur permet de développer indépendamment leurs modes d'étatisations. Dans cette phase antérieure, un État cherche, si l'on veut, le droit de s'engager dans les politiques interétatiques, un droit supposant en toile de fond un rapport global de souveraineté étatique.

Il est important de souligner que ce rapport de reconnaissance réciproque d'égalité et de liberté dépolitise les caractéristiques fondamentales de la vie commune à la base des rapports internationaux des États souverains. Par rapport à la lutte pour la reconnaissance politique d'Honneth, j'avance que la forme préalable de reconnaissance réciproque, qui rend possible cette lutte, dépend fondamentalement de la dépolitisation des caractéristiques fondamentales de nos pratiques mondiales. La lutte a pour but une reconnaissance éthique. 
Ceci est le point central que j'oppose à Honneth. Pour être reconnu comme un participant aux relations entre États souverains, il faut être constitué comme un acteur d'un certain type au sein de cette pratique interprétée éthiquement et non pas simplement politiquement. Cet acteur doit avoir un ensemble spécifique de libertés fondamentales. Pour le dire pragmatiquement, les libertés (ou droits) fondamentales sont : la liberté de chaque État souverain de développer ses propres politiques internes sans ingérence des autres États et le droit de chaque État de poursuivre sa propre politique étrangère. Les règles (dont une grande part est incorporée dans le droit international) qui constituent les États dans ces rapports étatiques sont des éléments strictement non négociables du point de vue des participants. Ceux-ci n'ont donc pas leur place dans la négociation ou la contestation politique. Ils n'ont pas leur place dans la contestation politique, car ils sont essentiels à l'identité de ces acteurs. En un sens, l'érosion ou la disparition (par un processus politique par exemple) de ces règles de souveraineté ferait que le rapport de souveraineté ne serait plus ce qu'il est, et que les États souverains ne seraient plus ce type d'acteurs que nous connaissons (et tels qu'eux-mêmes se connaissent). Être un État dans ce rapport, c'est être un acteur pour qui la souveraineté est éthiquement fondamentale. Ces règles consacrent la valeur éthique fondamentale de l'ensemble de cette pratique.

Il faut noter que cette base constitutive est valable pour toutes pratiques sociales (jeux, entreprises, églises, clubs, etc.) bien que les règles qui nous donnent l'essence individuelle de chaque pratique soient spécifiques à la pratique (le football est une pratique différente de la souveraineté). Toutes les pratiques possèdent un ensemble de règles, explicites ou non, qui précisent qui doit être reconnu comme participant de bonne foi ainsi que ce qui est autorisé ou non afin de rester dans le jeu. Pour être un participant, il faut être reconnu par les autres participants comme comprenant, acceptant et agissant en accord avec ces règles fondamentales. Prises ensemble, ces règles sont des règles constituantes parce qu'elles éclairent la « constitution » d'une pratique donnée. Dans toute pratique, il peut y avoir des différends entre participants sur l'interprétation de certaines parties de sa constitution. Lorsque cela se produit, on peut dire que les composants de la constitution ont été politisés. Mais du point de vue de ceux qui participent à la constitution d'une pratique quelconque, il existe une manière très concrète de mettre un élément constitutif à l'agenda politique. C'est ce que nous entendons lorsque nous décrivons ces règles de base comme élément constitutif. Le terme se réfère aux règles sociales par lesquelles les participants constituent l'autre comme le type d'acteur qu'ils apprécieraient d'être. La constitution définit le noyau éthique de toute pratique.

Revenons à notre exemple des États souverains. Une fois qu'un État a obtenu une reconnaissance comme État souverain libre, les autres États souverains qui participent aux relations internationales lui indiquent qu'ils le 
reconnaissent comme ayant une autonomie sur un ensemble de choses. Cette autonomie, conférant les « règles du jeu », se retrouve retirée du champ de la controverse. À partir de ce moment, l'État peut se concentrer sur l'élaboration pour sa population d'éléments de vie en commun, sans avoir à se battre sans cesse pour sa reconnaissance. Une fois cette forme de "reconnaissance » atteinte, l'État possède une base dépolitisée sûre à partir de laquelle il peut poursuivre ses projets.

Si nous passons maintenant à la deuxième grande pratique des relations internationales, celle de la société civile mondiale (SCM), nous pouvons discerner un schéma similaire de reconnaissance mutuelle entre individus, hommes et femmes, à l'intérieur des États, des communautés, auxquels ils appartiennent. Là encore, nous trouvons des acteurs, dans ce cas des individus, qui s'accordent les uns les autres un ensemble de droits fondamentaux de manière égalitaire. Là encore, les droits qu'ils s'accordent mutuellement n'existent que dans la mesure où les titulaires de ces droits se reconnaissent comme ayant de tels droits. Les droits ne préexistent pas à l'acte de reconnaissance mutuelle des acteurs engagés dans une relation normative. Je ne peux obtenir les droits d'une personne libre que dans la mesure où les personnes libres m'accordent de tels droits. Ma liberté dépend d'une reconnaissance donnée librement au sein d'un rapport de droits. Je ne peux forcer ce type de reconnaissance chez des personnes en les menaçant d'une arme à feu. La force, comprise de la sorte, n'est qu'un concept physique et non un concept normatif.

Là encore, quand je recherche cette forme de reconnaissance, ce pour quoi je me bats d'abord n'est ni la reconnaissance de ma philosophie particulière ou de mon mode de vie, ni le respect de ce que j'ai fait de ma vie, de mes valeurs culturelles et de mes réalisations, mais le respect d'un droit - mon droit d'avoir une vie et les droits qui me permettent de choisir comment vivre cette vie. Les droits sont des relations normativement soutenues, des relations supposant une réciprocité et, comme je voudrais le suggérer ici à la suite de Hegel, supposant une réciprocité sous forme de reconnaissance mutuelle. Je recherche, auprès des autres participants à la pratique des détenteurs de droits, la reconnaissance que je suis le type de personne qui a le droit de construire pour ellemême un projet de vie. Je demande la reconnaissance de ceci, même s'il s'avère que mon projet de vie n'est pas respecté, admiré ou cherché à être copié par les autres. En fait, d'autres pourraient même bien mépriser la vie que je me choisis.

Il est important de noter ici que lorsque j'obtiens une reconnaissance comme titulaire de droits individuels, il y a une dépolitisation importante d'un aspect de ma vie vis-à-vis des autres participants à cette pratique. Quand les autres participants à la SCM me reconnaissent comme possédant cet ensemble fondamental de droits de l'Homme, ils ne le reconnaissent que dans les limites 
de cette relation - énoncée dans une constitution ou un ensemble de règles de base -, et ces droits deviennent donc inappropriés comme source de différends politiques. Ils ont été, pour ainsi dire, sortis de la sphère politique. Une fois reconnu comme titulaire de droits, je peux alors les déployer pour construire ma vie au sein de la SCM, confiant dans le fait qu'ils ne seront pas encore et encore repolitisés. La dépolitisation de cet aspect de notre vie commune dans les relations de la SCM est pour partie ce que nous entendons lorsque nous réclamons des droits pour nous-mêmes.

Ce qui découle de l'analyse d'une dépolitisation implicite des rapports des États souverains et de la société civile mondiale est que même si la lutte pour accéder à son propre mode de vie est importante pour n'importe quel État, elle ne l'est pas autant que la défense de la pratique de la souveraineté. La pratique de la souveraineté offre un statut constituant et c'est en vertu de cette signification éthique qu'elle permet à une lutte pour la reconnaissance plus superficielle, c'est-à-dire politique, d'être en premier lieu.

De même, dans la SCM on pourrait reconnaitre que, malgré l'importance d'une lutte menée par les titulaires de droits individuels pour que leur mode de vie (chrétien, musulman, hindou, bouddhiste, etc.) soit respecté, cette lutte n'est pas aussi importante que la sécurisation même de la SCM, la sécurisation des relations constituant ces agents comme ayant le droit de construire leur propre vie sous réserve de la contrainte élémentaire et donc non-politisable des droits donnés entre les titulaires de ceux-ci. Si nous nous voyons mutuellement comme des titulaires de droits au sein de la SCM, la détention de droits ne peut être un sujet de litige politique. Prétendre le contraire revient à traiter la SCM comme une pratique possédant un statut éthique fondamental pour ses membres, mais aussi comme ayant un statut purement politique pouvant être renégocié et modifié à tout moment, à chaque fois que la constellation des forces politiques modifie son cours. Les pratiques peuvent changer dans leurs contours, mais pas en leur cœur ou leur constitution.

Tout ceci nous mène à la question d'une candidature à une adhésion. Du point de vue des entités qui ne sont pas encore des participants à part entière, mais qui cherchent à le devenir, l'élément important à noter n'est pas la lutte pour tel ou tel mode de vie particulier (palestinien, tchétchène, ossète du sud, basque, etc.), mais la décision de souscrire aux éléments ou règles clés constitutifs de cette pratique. Le rapport de souveraineté suggère donc que l'obtention d'une reconnaissance comme État souverain signifie qu'en premier lieu on est en droit - ou qu'on a le droit - de poursuivre son propre mode de vie (sa propre forme de vie et culture). De même, pour les personnes ne participant pas encore à la pratique des droits de l'Homme, la quête d'une reconnaissance de son mode de vie n'est pas aussi importante que celle d'être en droit de choisir indépendamment son mode de vie. 
J'espère que l'argumentaire développé ici, qui décrit comment les États et les détenteurs de droits individuels se constituent comme des acteurs appréciant d'être parties prenantes des pratiques mondiales de la souveraineté et de la SCM -, éclairent les valeurs fondamentales qu'ils défendent. Ce que doivent défendre les participants (à la limite, mais seulement à la limite, par un recours à la force), ce sont les règles constitutives dépolitisées. En ce qui concerne les États souverains, les États membres de cette société doivent avoir pour engagement fondamental le maintien des règles constituant la souveraineté. En tant qu'États souverains, ils ne sont pas autorisés à admettre l'émergence de règles qui les remettraient en cause. Non pas que cela soit impossible, mais cela enlèverait tout sens à cette entreprise. Repousser les règles constitutives revient à refuser de jouer le jeu. Un tel défi, si les États le poursuivaient, compromettrait leur propre position d'entités souveraines au sein du rapport de souveraineté, alors que c'est celle-ci qui leur donne autorité chez eux. De même, les titulaires de droits dans la SCM doivent agir fermement de manière à défendre les règles présentes dans la SCM car elles les constituent comme titulaires de droits. Quels que soient les modes de vie que l'on est en droit de choisir, il s'agit de décisions politiques importantes mais qui restent secondaires par rapport à la préservation des droits constituant la pratique.

Pour conclure, je dois souligner que l'élément central à la compréhension de la reconnaissance mutuelle des États et des individus dans ces deux pratiques est l'analyse de ceux-ci comme des touts sociaux (ou des «pratiques sociales totales » en référence à Mauss). Chaque pratique dans laquelle certains acteurs sont constitués comme participants est une totalité signifiante dont les significations guident l'action, qu'il s'agisse d'États souverains, de détenteurs de droits individuels ou de joueurs de football. L'élément central avancé ici est que les formes les plus fondamentales de reconnaissance réciproque dans les rapports mondiaux sont issues de ces deux pratiques sociales. 